\title{
Femtosecond laser crystallization of amorphous Ge
}

Omer Salihoglu, Ulas, Kürüm, H. Gul Yaglioglu, Ayhan Elmali, and Atilla Aydinli

Citation: Journal of Applied Physics 109, 123108 (2011);

View online: https://doi.org/10.1063/1.3601356

View Table of Contents: http://aip.scitation.org/toc/jap/109/12

Published by the American Institute of Physics

\section{Articles you may be interested in}

Femtosecond laser-induced periodic surface structures

Journal of Laser Applications 24, 042006 (2012); 10.2351/1.4712658

Laser crystallization and structuring of amorphous germanium

Applied Physics Letters 70, 3570 (1998); 10.1063/1.119236

Semiconducting and other major properties of gallium arsenide Journal of Applied Physics 53, R123 (1998); 10.1063/1.331665

Effect of medium range order on pulsed laser crystallization of amorphous germanium thin films Applied Physics Letters 108, 221906 (2016); 10.1063/1.4953153

Phase transformation mechanisms involved in excimer laser crystallization of amorphous silicon films Applied Physics Letters 63, 1969 (1998); 10.1063/1.110617

\section{Scilight}

Sharp, quick summaries illuminating the latest physics research

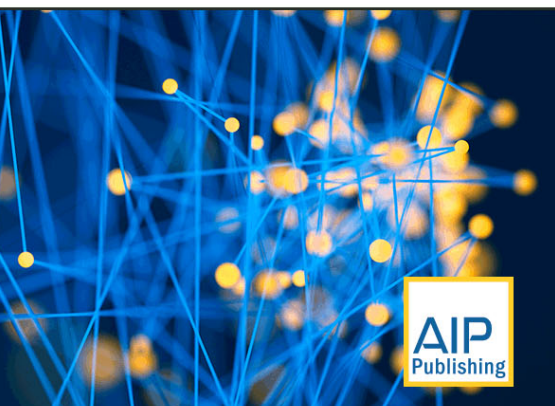




\title{
Femtosecond laser crystallization of amorphous Ge
}

\author{
Omer Salihoglu, ${ }^{1, a)}$ Ulaş Kürüm, ${ }^{2}$ H. Gul Yaglioglu, ${ }^{2}$ Ayhan Elmali, ${ }^{2}$ and Atilla Aydinli ${ }^{1}$ \\ ${ }^{1}$ Physics Department, Bilkent University, Ankara 06800, Turkey \\ ${ }^{2}$ Department of Engineering Physics, Ankara University, 06100 Ankara, Turkey
}

(Received 24 February 2011; accepted 12 May 2011; published online 23 June 2011)

\begin{abstract}
Ultrafast crystallization of amorphous germanium (a-Ge) in ambient has been studied. Plasma enhanced chemical vapor deposition grown a-Ge was irradiated with single femtosecond laser pulses of various durations with a range of fluences from below melting to above ablation threshold. Extensive use of Raman scattering has been employed to determine post solidification features aided by scanning electron microscopy and atomic force microscopy measurements. Linewidth of the Ge optic phonon at $300 \mathrm{~cm}^{-1}$ as a function of laser fluence provides a signature for the crystallization of a-Ge. Various crystallization regimes including nanostructures in the form of nanospheres have been identified. (C) 2011 American Institute of Physics. [doi:10.1063/1.3601356]
\end{abstract}

\section{INTRODUCTION}

Crystallization of amorphous materials can be achieved with different techniques such as furnace and rapid thermal annealing, ${ }^{1}$ ion beam treatment, ${ }^{2}$ pulsed and cw laser annealing. ${ }^{3-5}$ Laser induced crystallization of amorphous structures reduce thermal budget and provide crystallization of predetermined patterns down to sizes only limited by diffraction of light allowing space selective writing of crystalline structures in amorphous matrices.

Many types of lasers have been used in the past to crystallize amorphous semiconductors. ${ }^{3,4}$ Recent advances in ultrafast lasers in the femtosecond time regime allows us to exploit solid and liquid state crystallization of amorphous semiconductors in the ultrafast regime. Unlike nanosecond or picosecond time regimes, energy deposition in the femtosecond time regime which is faster than electron-phonon interaction times can lead to excitation of electron densities beyond those required for lattice stability. It has been shown that crystalline $\mathrm{Si}, \mathrm{Ge}$, and GaAs show nonthermal melting ${ }^{5,6}$ in the subpicosecond time scale upon irradiation with femtosecond laser pulses while thermal melting occurs only at longer time scales. This has been confirmed with ultrafast x-ray diffraction as well as light scattering and reflection measurements. ${ }^{5,7}$ Thus, the energy deposited by ultrafast lasers into the semiconductor is initially stored in the electronic system. If the intensity of the laser is high enough, it may be possible to excite high density of electrons to cause dissolution of the solid up to a thickness of a few tens of nm. As the energy is transferred to the lattice with electron-phonon interaction, nonthermal melting regime ends and a hot liquid develops. Once the rate of energy deposition drops sharply, cooling process starts to take place at a rate that is determined by thermal parameters of the semiconductor lasting up to several hundred nanoseconds. ${ }^{8}$ The rate of cooling determines the crystallinity of the material and may result in epitaxial layers with appropriate seeding as well as in microcrystals

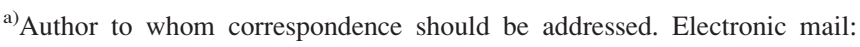
omersalihoglu@yahoo.com.
}

and nanocrystals. Competition between nucleation and growth rate determines grain sizes both of which depend on the background temperature. High growth rate together with low nucleation rate results in few large nanocrystals, while slow growth rate together with high nucleation rate results in many small nanocrystals. ${ }^{8}$ The possibility of creating nanocrystals with ultrafast lasers is exciting. However, controlling the density and size distribution of the nanocrystals is a challenge. Number of pulses and fluence per pulse can change the density of the nanocrystals. Alternatively, single crystal seeds can be used to control location and the density of the nanocrystals. ${ }^{9}$

Previous work shows that femtosecond laser irradiation of single crystal Ge wafers can create microstructures with attached nanoclusters that show strong photoluminescence (PL) in the visible region. ${ }^{10}$ Critical combination of pulse duration and pulse energy is required to cause melting or ablation of the crystalline germanium surface with femtosecond laser irradiation. ${ }^{5}$ Interaction of femtosecond lasers with semiconductors has recently produced interesting surface structures. In particular, laser pulses of a $130 \mathrm{fs}$ incident on single crystal germanium $(\mathrm{Ge})$ in $\mathrm{SF}_{6}$ environment has resulted in ordered arrays of cones with nanospikes. ${ }^{11}$ Thin amorphous germanium films deposited on low thermal conductivity quartz or glass has been studied during nanosecond irradiation with real time reflectivity measurements both from the front and back surfaces which show formation and evolution of a liquid melt. Both in picosecond and nanosecond time scales, increasing fluence increases the melt duration as well as the formation of a secondary melt during solidification due to the heat released upon solidification (recalescence process). ${ }^{12}$ Buried melts ${ }^{13}$ and in the picosecond time regime, surface initiated solidification upon complete melting of the film at high fluences have also been observed. ${ }^{14}$ In this study, we report on the interaction of femtosecond pulses of varying pulse durations and fluences with amorphous germanium thin films. The objective of this study is the identification of structural changes that occur on the surface of amorphous Ge upon irradiation with femtosecond lasers of various durations. Optical and electron microscopy, 
micro Raman spectroscopy and atomic force microscopy is used to identify the origins of the structures observed.

\section{EXPERIMENTAL}

Plasma enhanced chemical vapor deposition was used to grow amorphous $\mathrm{Ge}$ (a-Ge) film on silicon wafers. Full 3 in. double side polished silicon wafers were used. Growth of a-Ge film was carried out in PlasmaLab $8510 \mathrm{C}$ reactor at $350{ }^{\circ} \mathrm{C}$ and the process was carried out under the pressure of 1 Torr and RF power of $12 \mathrm{~W}$. Flow rate was $200 \mathrm{sccm}$ for $\mathrm{GeH}_{4}(\% 2 \mathrm{in} \mathrm{He})$. Final thickness of the germanium layer was about $375 \mathrm{~nm}$. Sample was cleaved into $1 \mathrm{~cm}$ by $1 \mathrm{~cm}$ pieces for different laser irradiation sequences.

A Ti:sapphire femtosecond pulsed laser-amplifier system (Spectra Physics Tsunami-Spitfire Pro XP) with $1 \mathrm{kHz}$ repetition rate and $800 \mathrm{~nm}$ wavelength was used for the experiments. The energy per pulse for the experiments was adjusted to $30 \mu \mathrm{J}$. Pulse duration of the system was changed to $40,80,120$, and $160 \mathrm{fs}$ via compressor delay in the amplifier. A three-axis computer controlled motorized translational stage was used to illuminate different positions on the samples as well as to alter the fluences at the illuminated spots. The sample was mounted on the motorized stage and placed at the focal point of the laser. Fluence in $\mathrm{mJ} / \mathrm{cm}^{2}$ was adjusted by moving the sample away from the focal point of the lens. Translational stage was programmed such that after each line scan, motorized stage moves one step away from the optimum focal point along the optical axis to change the spot size of the focused laser beam and one step vertically to start a new line scan on a fresh a-Ge surface. This method creates irradiated spots with different fluences. The spot sizes of the laser at position of the sample were measured by using a razor blade technique with an accuracy of $\pm 5 \mu \mathrm{m} .{ }^{15}$ The minimum laser spot size on the sample was $30 \mu \mathrm{m}$. We check the beam profile with a beam spot analyzer before we use the beam and find it to be Gaussian. The beam size on the 1 inch lens is not more than $3 \mathrm{~mm}$ in diameter reducing spatial inhomogenities. The overall error in the measured energy density is less than $1 \%$. Scan step sizes of 0.50 and $0.25 \mathrm{~mm}$ were used in backwards and forwards directions, respectively and the horizontal scan speed was $400 \mathrm{~mm} / \mathrm{s}$. Movement of the translational stage created 30 different irradiation lines containing separate laser treated spots in amorphous germanium which were irradiated with fluences ranging from 18 to $2000 \mathrm{~mJ} / \mathrm{cm}^{2}$. Each line contains 25 identical laser spots which are irradiated with the same fluence of the laser with center to center seperation of $400 \mu \mathrm{m}$. Although only one spot is enough for measurements, many spots give us a chance to check homogeneity of the laser beam from one spot to another. All laser treatments have been done under ambient temperature and pressure. Laser irradiated samples were analyzed with optical microscope, scanning electron microscopy (SEM), atomic force microscopy (AFM), and Raman scattering.

\section{RESULTS AND DISCUSSION}

For irradiated spots with fluences lower than $26 \mathrm{~mJ} / \mathrm{cm}^{2}$, no sign of morphological change under inspection with opti-

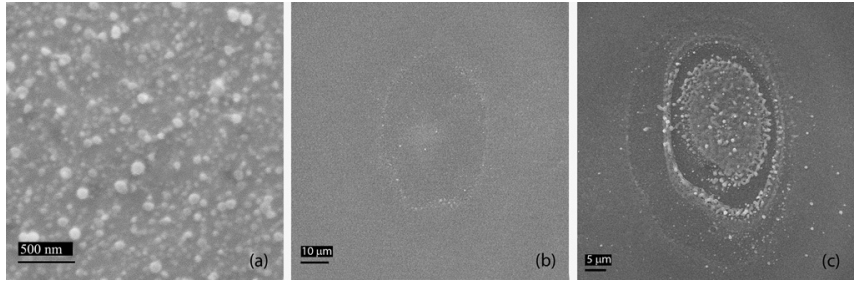

FIG. 1. Scanning electron microscope image of a-Ge surfaces irradiated with single pulses of $40 \mathrm{fs}$ duration and (a) $140 \mathrm{~mJ} / \mathrm{cm}^{2}$, (b) $340 \mathrm{~mJ} / \mathrm{cm}^{2}$, (c) $2000 \mathrm{~mJ} / \mathrm{cm}^{2}$.

cal or scanning electron microscopy (SEM) and atomic force microscopy (AFM) or of crystallization by Raman scattering was observed. As the fluence is increased beyond $26 \mathrm{~mJ} /$ $\mathrm{cm}^{2}$, samples start to show changes on the surface of amorphous Ge that lead to the growth of a circular spot which finally evolves into a well defined crater, visible by optical microscope, SEM, and AFM. Morphological changes upon laser irradiation as observed with SEM is summarized in Fig. 1. At laser fluence of $140 \mathrm{~mJ} / \mathrm{cm}^{2}$ with 40 fs pulse duration, surface roughness accompanied by spherical nanoparticles is observed, Fig. 1(a). A circular spot with weak contrast is also observed under optical microscope for this sample. As the laser fluence is increased, surface roughness further increases covering a larger area. At $340 \mathrm{~mJ} / \mathrm{cm}^{2}$, a clear sign of damage is visible featuring a large oval spot on the order of $20 \mu \mathrm{m}$ in diameter, Fig. 1(b). At the very highest laser fluence of $2000 \mathrm{~mJ} / \mathrm{cm}^{2}$ used in this study, an oval spot accompanied with an oval track encircling the central spot is observed, Fig. 1(c). In all cases, nanoparticles ranging in size from a few tens of nanometers $(\mathrm{nm})$ to several hundred $\mathrm{nm}$ accompany the spots. SEM observations suggest that as laser fluence is increased beyond required for melting and higher amounts of energy is deposited in the samples superheated liquids are generated that solidify into droplets of a few micrometers in size, reminiscent of splashing liquids, Fig. 1(c).

To overcome the limited information obtained from SEM observations, we also studied the same samples with AFM. AFM provides topographic information in three dimensions as well as allowing for measurement of sizes and length scales in all three dimensions. We also used surface profile measurements to confirm the threshold for ablation. The lowest fluence where there is nanosphere formation on the irradiated surface occurs is $110 \mathrm{~mJ} / \mathrm{cm}^{2}$ and the lowest fluence where ablation occurs is $210 \mathrm{~mJ} / \mathrm{cm}^{2}$. The surface structures that form at $140 \mathrm{~mJ} / \mathrm{cm}^{2}$, are shown in Fig. 2(a). Spherical nanoparticles ranging in size from 50 to $150 \mathrm{~nm}$ are clear evidence of melting followed by rapid solidification. Increasing the laser fluence increases the area over which these nanoparticles are formed as well as increasing the average size and density of spherical nanoparticles. The ablation from the surface is clearly observed on the sample that is irradiated with $340 \mathrm{~mJ} / \mathrm{cm}^{2}$, Fig. 2(b). The crater and the associated nanoparticles are visible under optical microscope as a dark spot in the middle of a larger circular area. AFM shows a well delineated circular crater with material piled up at the edges of the crater, Fig. 2(b). The pileup 


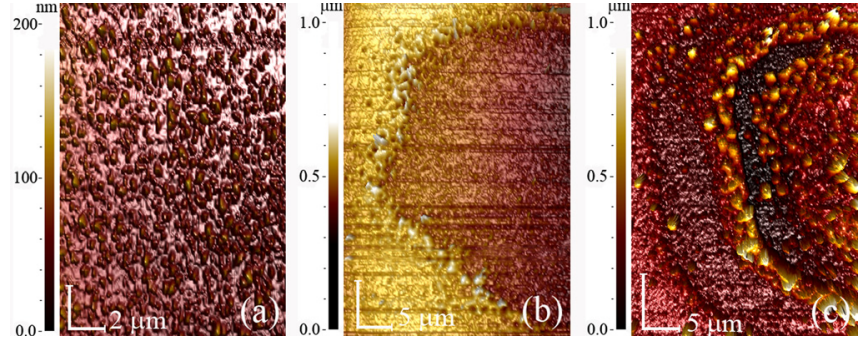

FIG. 2. (Color online) Atomic force microscope images of a-Ge surfaces irradiated with single pulses of $40 \mathrm{fs}$ duration with energy densities of (a) $140 \mathrm{~mJ} / \mathrm{cm}^{2}$, (b) $340 \mathrm{~mJ} / \mathrm{cm}^{2}$, (c) $2000 \mathrm{~mJ} / \mathrm{cm}^{2}$. Note that Nanosphere formation starts at $110 \mathrm{~mJ} / \mathrm{cm}^{2}$. Increasing laser fluence results in the onset of ablation at $210 \mathrm{~mJ} / \mathrm{cm}^{2}$.

suggests that the temperature of the liquid has risen above the melting point of a-Ge. Increasing the laser fluence further increases the ablated area and makes it deeper, Fig. 2(c). At the highest laser fluence, the crater displays several ridges, suggestive of highly perturbed melt and solidification. Line profile of the AFM data gives valuable information on the size and distribution of the nanoparticles observed on the samples. Two such line scans are shown for samples irradiated with 140 and $340 \mathrm{~mJ} / \mathrm{cm}^{2}$, Fig. 3. Nanoparticles with dimensions of few tens of $\mathrm{nm}$ are easily discernible in Fig. 3(a). At higher fluences, the ablation of the laser spot leaves behind a shallow crater, Fig. 3(b). We find that the depth of the crater for laser fluence of $340 \mathrm{~mJ} / \mathrm{cm}^{2}$ is approximately $100 \mathrm{~nm}$.

In order to complement the topographic information supplied by SEM and AFM observations and understand the composition and crystallinity of the irradiated surfaces, we performed Raman scattering experiments. Raman spectroscopy provides one of the best ways of probing crystallization properties of the laser modified surface. We used a Horiba LabRAM HR microRaman spectrometer to observe the Raman signal using the $532.1 \mathrm{~nm}$ laser line, with a spot size

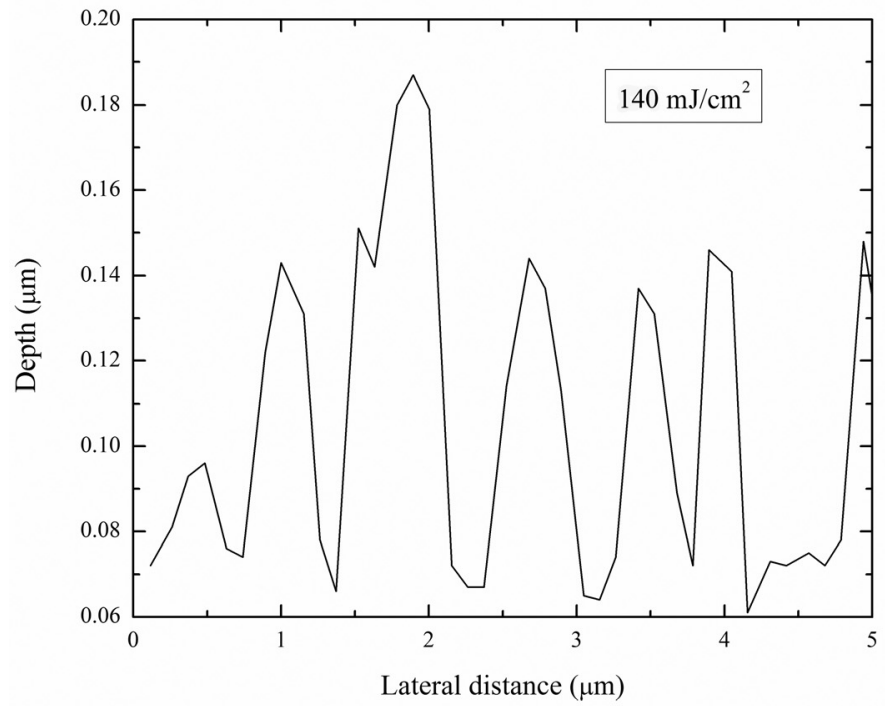

(a)

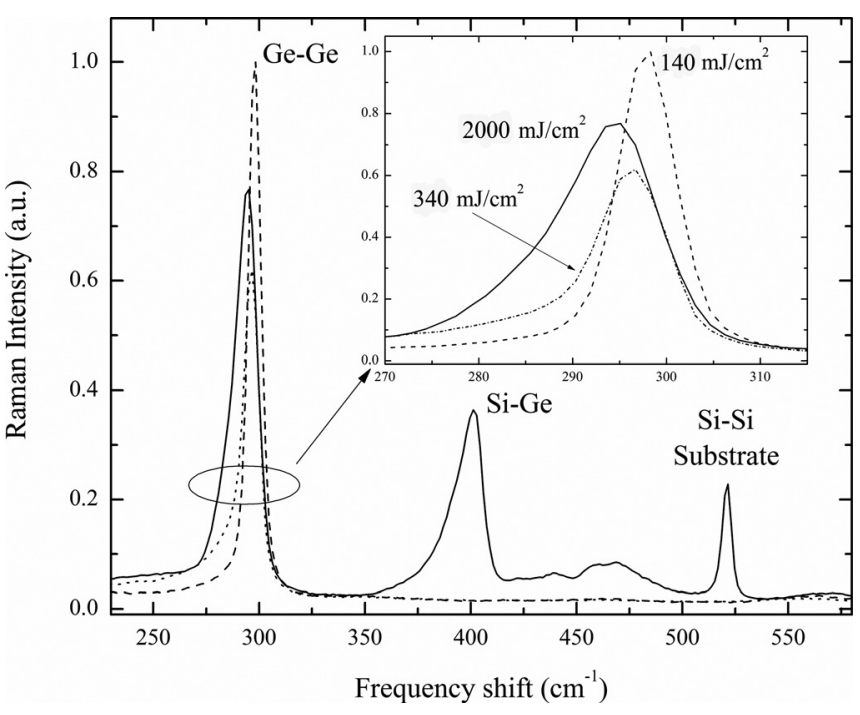

FIG. 4. Raman spectra of a-Ge irradiated with single pulses of 140, 340, and $2000 \mathrm{~mJ} / \mathrm{cm}^{2}$. Spectra at lower laser fluences display a peak at around $300 \mathrm{~cm}^{-1}$, indicative of the Ge optic phonon while the spectrum at the largest fluence shows marked evidence of SiGe alloy formation with line near $401 \mathrm{~cm}^{-1}$. The inset compares the line widths of the Ge optic phonon lines for all three irradiated spots.

of 4-5 $\mu \mathrm{m}$. Figure 4 shows Raman spectra of several irradiated spots irradiated with fluence of 140,340 , and $2000 \mathrm{~mJ} /$ $\mathrm{cm}^{2}$. In contrast with as grown a-Ge where only a broad peak below $300 \mathrm{~cm}^{-1}$ is observed representative of the phonon density of states, laser irradiated surfaces show strong peaks at around $300 \mathrm{~cm}^{-1}$, indicative of crystallization of $\mathrm{Ge}$ for all laser fluences. In the case of the sample with the highest irradiation dose of $2000 \mathrm{~mJ} / \mathrm{cm}^{2}$, there are additional features in the Raman spectrum. These consist of a peak at 401 $\mathrm{cm}^{-1}$ due to $\mathrm{SiGe}$, a broad peak at $469 \mathrm{~cm}^{-1}$ due to stretching modes of Si-Si bonds and a sharp peak at $520 \mathrm{~cm}^{-1}$ due to the substrate. ${ }^{16}$ These peaks clearly indicate that the melt front has reached the substrate forming alloys of $\mathrm{Ge}$ with $\mathrm{Si}$

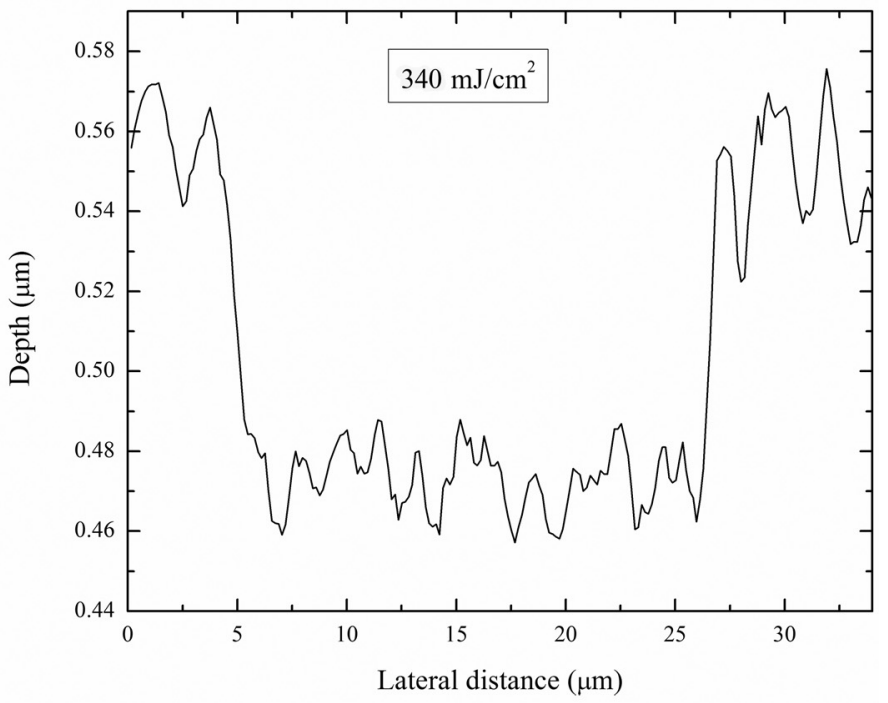

(b)

FIG. 3. Line profile of irradiated surfaces irradiated with (a) $140 \mathrm{~mJ} / \mathrm{cm}^{2}$ and (b) $340 \mathrm{~mJ} / \mathrm{cm}^{2}$ obtained with AFM. The height of the particles in (a) is a measure of the size of the nanospheres observed. 




FIG. 5. Raman signal from $97 \mathrm{~mJ} / \mathrm{cm}^{2}$ laser irradiated spot shows 5.75 $\mathrm{cm}^{-1}$ Raman width and $297.9 \mathrm{~cm}^{-1}$ Raman peak position. Inset shows germanium optic phonon line from the center of the laser irradiated spots at selected fluences with pulse duration of $40 \mathrm{fs}$.

substrate. As the laser pulse is absorbed in amorphous $\mathrm{Ge}$, a melt forms in the subpicosecond time regime reaching down to the substrate and melting the substrate. AFM images of the solidified surface suggests that a highly perturbed melt possibly under the influence of hydrodynamic effects undergoes rapid solidification.

It is interesting to study the Raman shift and the linewidth of the Ge optic phonon line when the laser fluence is increased, Fig. 4, inset. When compared with bulk Ge phonon line at $300.6 \mathrm{~cm}^{-1}$ with linewidth of $4 \mathrm{~cm}^{-1}$, linewidth of $7.0 \mathrm{~cm}^{-1}$ for the peak at $297.5 \mathrm{~cm}^{-1}$ for fluence of 140 $\mathrm{mJ} / \mathrm{cm}^{2}$ increases to $8.6 \mathrm{~cm}^{-1}$ with peak shifted down to $296.3 \mathrm{~cm}^{-1}$ for fluence of $340 \mathrm{~mJ} / \mathrm{cm}^{2}$. Linewidths larger than bulk values may be due to several factors including formation of defects during solidification, large variation in nanocrystal sizes and phonon confinement in nanocrystals. ${ }^{17}$ The Ge optic phonon line redshifts down to $294.5 \mathrm{~cm}^{-1}$, broadens to $11 \mathrm{~cm}^{-1}$ and becomes assymmetric for the sample irradiated with the highest laser fluence at $2000 \mathrm{~mJ} / \mathrm{cm}^{2}$, indicating large size distribution with a significant percentage of fine sized nanocrystals. The associated tensile stress increases upon solidification after irradiation with high fluence of $2000 \mathrm{~mJ} / \mathrm{cm}^{2}$ as suggested by a redshift of approximately $6 \mathrm{~cm}^{-1}$ from the bulk Ge phonon line. Using the approach of Fang et al., ${ }^{18}$ we estimate the tensile stress as $1.45 \%$.

In order to determine the crystallization threshold and observe the Raman peak with the smallest linewidth, systematic study of all samples was carried out and samples were irradiated with a large number of fluences. Figure 5 shows Raman spectra from a laser irradiated spot with $97 \mathrm{~mJ} / \mathrm{cm}^{2}$ and $40 \mathrm{fs}$ pulse duration. The inset of the figure shows typical germanium optic phonon line from the center of laser crystallized spots for fluences between 30 to $97 \mathrm{~mJ} / \mathrm{cm}^{2}$. It is clear that we observe crystallization of Ge at these energy densities, which are smaller than the laser fluence for the onset of nanosphere formation are $\left(110 \mathrm{~mJ} / \mathrm{cm}^{2}\right)$ and the

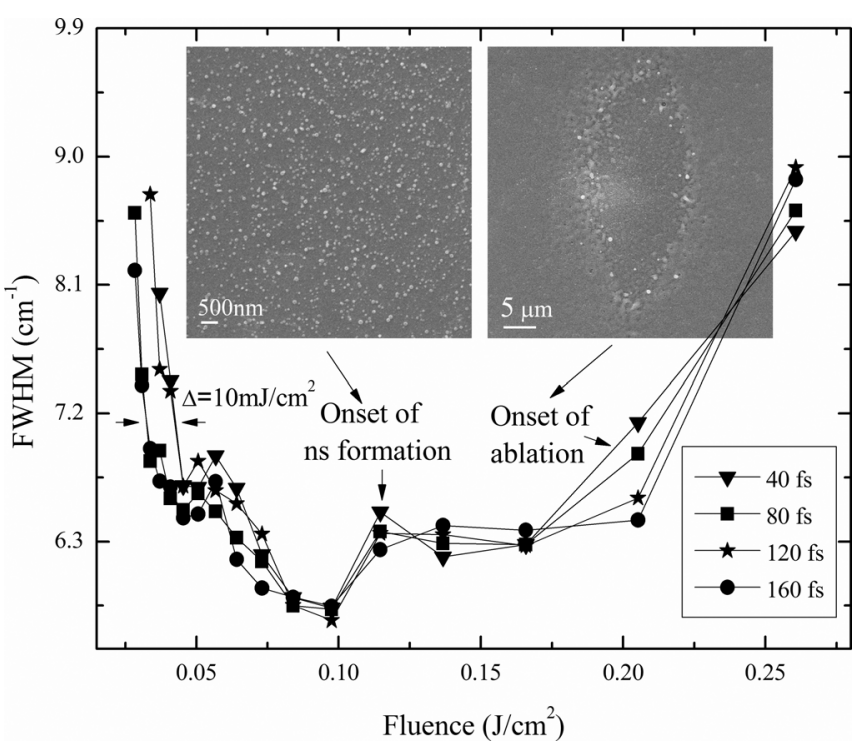

FIG. 6. FWHM of the Ge-Ge optic phonon line as a function of single shot laser fluence. Laser at $800 \mathrm{~nm}$ with 40, 80,120, and $160 \mathrm{fs}$ pulse width was used to irradiate the surface of the amorphous germanium layer. Laser fluence for best crystallization with the narrowest Raman line occurred at the laser fluence of $100 \mathrm{~mJ} / \mathrm{cm}^{2}$ for the pulse duration of $40 \mathrm{fs}$. Two insets shows SEM images of on sets for nanostructure formation and ablation.

onset of ablation $\left(210 \mathrm{~mJ} / \mathrm{cm}^{2}\right)$. As expected, a-Ge displays a weak and broad Raman peak reflective of the germanium density of states. As fluence of the laser beam increases, spectra display typical $\mathrm{Ge}-\mathrm{Ge}$ optic phonon line close to 300 $\mathrm{cm}^{-1}$. A broad but localized peak centered around 297.0 $\mathrm{cm}^{-1}$ appears at the lowest fluence of $30 \mathrm{~mJ} / \mathrm{cm}^{2}$ used in this study. As the annealing laser energy density increases, the linewidth decreases and the peaks shift toward the bulk Ge value for the optic phonon reaching $298.0 \mathrm{~cm}^{-1}$ at $97 \mathrm{~mJ} /$ $\mathrm{cm}^{2}$. However, even when the smallest linewidth is reached and the symmetry of the line is restored, there still remains a small amount of redshift $\left(2.6 \mathrm{~cm}^{-1}\right)$ of the Raman line when compared with the bulk value. This is indicative of tensile stress experienced by the crystallized zone by the surrounding amorphous matrix ${ }^{18}$ and has been estimated to be $0.60 \%$.

Figure 6 shows the full width at half maximum (FWHM) of the Ge-Ge optic phonon line for the 40, 80, 120 , and 160 fs pulse durations. We did not observe crystallization for laser energy densities below $26 \mathrm{~mJ} / \mathrm{cm}^{2}$ as determined by Raman scattering. As the laser energy density increases, the FWHM of the Ge optic phonon line decreases reaching a minimum at $100 \mathrm{~mJ} / \mathrm{cm}^{2}$. We note that this behavior is the same for all pulse durations. For laser fluences higher than $100 \mathrm{~mJ} / \mathrm{cm}^{2}$, the FWHM of the Ge-Ge mode increases. After $110 \mathrm{~mJ} / \mathrm{cm}^{2}$ nanoparticles appear on the surface of the germanium layer. SEM images in the inset of Fig. 6 show the surface morphology for the particular fluence. Size of the nanoparticles range between 20 to $80 \mathrm{~nm}$. These nanostructures are created upon rapid cooling and solidification of the germanium layer. While the size of nanospheres can be considered keeping in mind that optical penetration of the irradiation laser (@800 nm) in amorphous Ge is about $100 \mathrm{~nm}$, nonlinear thermal processes as well as the possible modification of optical penetration depth during irradiation 


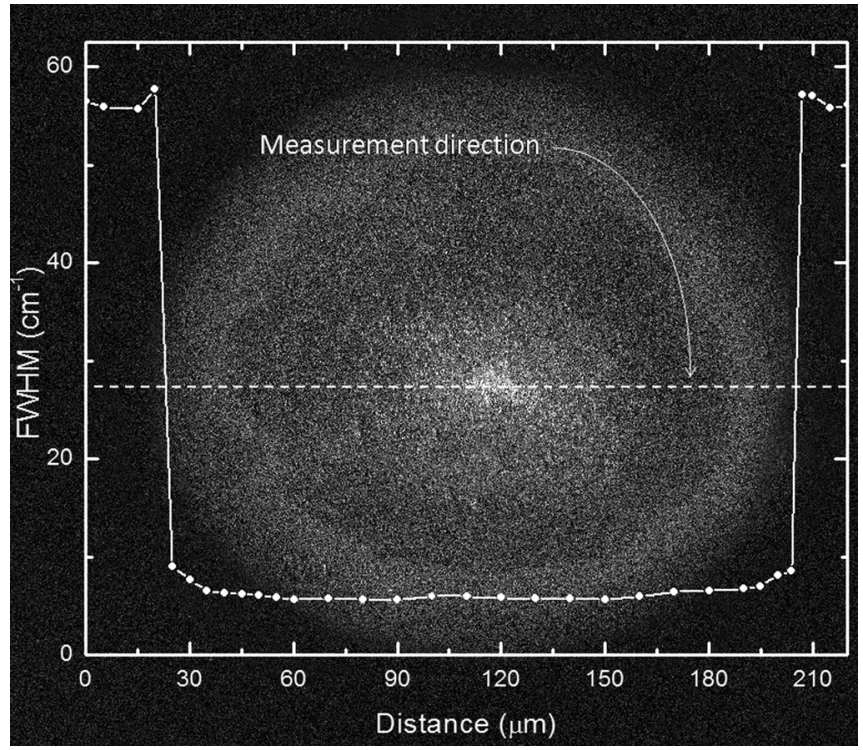

FIG. 7. SEM image and FWHM of the Raman signal along the centerline of the irradiation spot irradiated with $140 \mathrm{~mJ} / \mathrm{cm}^{2}$ and $40 \mathrm{fs}$ pulse duration.

needs to be taken into account for a complete picture, which is beyond the scope of this article. High fluences cause higher temperatures at the irradiated surface and lower cooling rates. ${ }^{19}$ The second inset shows a SEM image of laser irradiated spot with $210 \mathrm{~mJ} / \mathrm{cm}^{2}$ fluence where ablation of the germanium layer starts.

The linewidth and shift of the Raman frequency depends on several factors such as crystal size and defects, finite size effects, ${ }^{20,21}$ as well as stress. It is well known that tensile stress results in Stokes frequency to redshift, while compressive stress leads to blueshift. ${ }^{20}$ Group IV semiconductors such as $\mathrm{Si}$ and Ge broaden their Stokes linewidth and shift toward shorter wavenumbers when the crystal sizes become smaller than $5 \mathrm{~nm}^{21}$ Since it is well known that finite size effects cause broader Raman line widths, increase in the Raman linewidth beyond $100 \mathrm{~mJ} / \mathrm{cm}^{2}$, may be due to the formation of smaller nanocrystals upon solidification. ${ }^{21}$ Alternatively, high defect densities in the crystallized Ge may cause broader linewidth in Raman spectra. ${ }^{22}$ A TEM study on femtosecond laser ablation of amorphous silicon by Rogers et al. showed that ablation leaves behind defective crystalline material. ${ }^{23}$

Under our experimental conditions of $800 \mathrm{~nm}$ laser wavelength, $1 \mathrm{kHz}$ pulse rate and $400 \mathrm{~mm} / \mathrm{s}$ scan speed of the translational stage, the best crystallization with the narrowest Raman linewidth occurred at laser fluence of 100 $\mathrm{mJ} / \mathrm{cm}^{2}$. We have repeated this experiment for different pulse durations of 40,80, 120, and $160 \mathrm{fs}$; we did not observe significant differences in the Raman linewidth when the pulse durations were varied except at very low fluences. It seems that about $10 \mathrm{~mJ} / \mathrm{cm}^{2}$ less energy is required for crystallization to start for long pulse durations (120 and $160 \mathrm{fs}$ ) than at shorter pulses. At very low laser fluences, longer pulse durations possibly provide slower melting with lower melt temperatures with less perturbation and longer melt durations that leads to crystallization with lower defect densities.
Figure 7 shows the FWHM of the Raman signal along the irradiated spot and a SEM image of the same spot superimposed. The dashed line shows the Raman measurement direction. Both of them have the same scale in the measurement direction. Using a translational stage, the sample was moved along the measurement direction and Raman signal has been recorded to compare the crystallization quality along the laser irradiated spot. The FWHM of the GE optic phonon line obtained for each spot shows that inside the irradiated spot there is good crystallization but immediately outside the irradiated spot there is no crystallization. The FWHM of the Raman peak stays almost constant over the irradiated spot, suggesting uniform crystallization. We estimate that a transition takes place within a few $\mu \mathrm{m}$. Considering the total diameter of the irradiated spot, the relative magnitude of the transition region is negligible. This transition makes femtosecond laser irradiation a very usable tool to create space selected annealing. There is very little lateral penetration of the laser deposited energy. Once amorphous Ge melts, the melt front rapidly moves forward, faster than lateral heat flow, limited by low thermal conductivity of amorphous Ge. This is a sign that small spots with high aspect ratio can be crystallized in amorphous Ge suitable for many applications such as the introduction of nanocrystals and microcrystals in amorphous solar cells in order to tailor the absorption spectrum of the solar cell. While it is true that most of the energy is deposited within the optical penetration depth, it is also clear that thermal energy diffuses beyond this thickness. Furthermore, should the layer melt (nonthermal or thermal) during the pulse, optical penetration depth may be significantly modified.

\section{CONCLUSION}

In conclusion, we have investigated the effect of femtosecond laser irradiation on the solidification of amorphous Ge films. Laser pulses with pulse durations of 40, 80, 120, and 160 fs with various fluences were used for the irradiations. We observe a very intense Ge-Ge Raman peak around $300 \mathrm{~cm}^{-1}$ which is an excellent indication of the crystallization. Thus, we have observed crystallization for all pulse durations and for fluences greater than $26 \mathrm{~mJ} / \mathrm{cm}^{2}$. Analysis of the Ge-Ge optic phonon mode width suggest that for the same fluence longer pulses lead to narrower linewidths which may be due to slower cooling rates. When laser fluence increases beyond $50 \mathrm{~mJ} / \mathrm{cm}^{2}$, different pulse durations do not change the Raman spectra. We did not observe crystallization below laser fluence of $26 \mathrm{~mJ} / \mathrm{cm}^{2}$. Narrowest linewith occurred at $100 \mathrm{~mJ} / \mathrm{cm}^{2}$ indicating that this is the laser fluence where best crystallization with least number of defects occur. For fluences higher than $100 \mathrm{~mJ} / \mathrm{cm}^{2}$, Raman peak position moves toward shorter wavenumbers and broadens. Laser pulses created very well defined irradiation marks on the amorphous germanium surfaces visible by optical microscopes for all fluences mentioned in this study. Inside the irradiated spots, in contrast with the observed GeGe Raman peak inside the irradiated spots, no Ge-Ge optic phonon line was observed immediately outside of the irradiated spot. This shows that there is very little lateral 
penetration of the laser deposited energy. We showed that femtosecond laser crystallization is an efficient and controlled method to crystallize amorphous germanium and it can be used to create a variety of nanostructures including nanospheres.

\section{ACKNOWLEDGMENTS}

The authors would like to thank Nanomagnetics Instruments, Ltd., for AFM measurements. This work was supported by EU Seventh Framework project Unam_Regpot (Grant No. 203953).

${ }^{1}$ A. Dana, S. Agan, S. Tokay, A. Aydinli, and T. G. Finstad, Phys. Status Solidi 4, 288 (2007).

${ }^{2}$ N. P. Stepina, A. V. Dvurechenskii, V. A. Armbrister, V. G. Kesler, P. L. Novikov, A. K. Gutakovskii, V. V. Kirienko, Zh. V. Smagina, and R. Groetzschel, Appl. Phys. Lett. 90, 133120 (2007).

${ }^{3}$ L. B. Ma, R. Song, Y. M. Miao, C. R. Li, Y. Du, Y. Q. Wang, and Z. X. Cao, Appl. Phys. Lett. 88, 093102 (2006).

${ }^{4}$ R. Huang, L. B. Ma, Y. Du, L. Gao, C. R. Li, C. L. Yu, J. P. Ye, and Z. X. Cao, Nanotechnology 19, 255402 (2006).

${ }^{5}$ A. Cavalleri, C. W. Siders, C. Rose-Petruck, R. Jimenez, C. Toth, J. A. Squier, C. P. J. Barty, K. R. Wilson, K. Sokolowski-tinten, M. H. Hoagen, and D. Linde, Phys. Rev. B 63, 193306 (2001).

${ }^{6}$ C. V. Shank, R. Yen, and C. Hirlimann, Phys. Rev. Lett. 50, 454 (1983).
${ }^{7}$ Rousse, C. Rischel, S. Fourmaux, I. Uschmann, S. Sebban, G. Grillon. Ph. Balcou, E. Forster, J. P. Geindre, P. Audebert, J. C. Gauthier, and D. Hulin, Nature 410, 65 (2001).

${ }^{8}$ F. Falk and G. Andrä, J. Cryst. Growth 287, 397 (2006).

${ }^{9}$ G. Nakagawa and T. Asano, J. Appl. Phys. 47, 3036 (2008).

${ }^{10}$ S. C. Jeoung, H. S. Kim, M. Park, J. Lee, and C. O. Park, J. Appl. Phys. 44, 5278 (2005).

${ }^{11}$ K. Nayak, M. C. Gupta, and K. W. Kolasinski, Nanotechnology 18, 195302 (2007).

${ }^{12}$ J. Siegel, J. Solis, and C. N. Afonso, Appl. Phys. Lett. 75, 1071 (1999).

${ }^{13}$ F. Vega, N. Chaoui, J. Solis, J. Armengol, and C. N. Afonso, J. Appl. Phys. 97, 103519 (2005).

${ }^{14}$ J. Siegel, J. Solis, C. N. Afonso, F. Vega, J. Bankmann, O. Martínez Sacristán, and K. Sokolowski-Tinten, J. Appl. Phys. 89, 3642 (2001).

${ }^{15}$ R. Skinner and R. E. Whitcher, J. Phys. E 5, 237 (1972).

${ }^{16}$ J. Olivares, P. Martin, A. Rodriguez, J. Sangrador, J. Jimenez, and T. Rodriguez, Thin Solid Films 358, 56 (2000).

${ }^{17}$ Bersani, P. P. Lottici, and X. Z. Ding, Appl. Phys. Lett. 72, 1 (1998).

${ }^{18}$ Y.-Y. Fang, J. Tolle, R. Roucka, A. V. G. Chizmeshya, and J. Kouvetakis, V. R. D'Costa, and J. Menéndez, Appl. Phys. Lett. 90, 061915 (2007).

${ }^{19}$ M. J. Jackson and G. M. Robinson, J. Achiev. Mater. Manuf. Eng. 22, 81 (2007).

${ }^{20}$ A. Wellner, V. Paillard, C. Bonafos, H. Coffin, A. Claverie, B. Schmidt, and K. H. Heinig, J. Appl. Phys. 94, 5639 (2003).

${ }^{21}$ S. K. Gupta and P. K. Jha, Solid State Commun. 149, 1989 (2009).

${ }^{22}$ K. Kitahara, K. Ohnishi, Y. Katoh, R. Yamazaki, and T. Kurosawa, Jpn. J. Appl. Phys. 42, 6742 (2003).

${ }^{23}$ M. S. Rogers, C. P. Grigoropoulos, A. M. Minor, and S. S. Mao, Appl. Phys. Lett. 94, 011111 (2009). 